\title{
The Association of Acquired T790M Mutation with Clinical Characteristics after Resistance to First-Line Epidermal Growth Factor Receptor Tyrosine Kinase Inhibitor in Lung Adenocarcinoma
}

\author{
Yen-Hsiang Huang, MD ${ }^{1}$ \\ Kuo-Hsuan Hsu, MD2,3 \\ Jeng-Sen Tseng, MD, PhD',4 \\ Kun-Chieh Chen, MD, PhD ${ }^{1}$ \\ Chia-Hung Hsu, MD' \\ Kang-Yi Su, PhD 5,6 \\ Jeremy J. W. Chen, $\mathrm{PhD}^{3}$ \\ Huei-Wen Chen, $\mathrm{PhD}^{7}$ \\ Sung-Liang $\mathrm{Yu}, \mathrm{PhD}^{5,6,8,9,10}$ \\ Tsung-Ying Yang, $\mathrm{MD}, \mathrm{PhD}^{1,4}$ \\ Gee-Chen Chang, MD, PhD'1,411
}

*A list author's affiliations appears at the end of the paper.

Correspondence: Gee-Chen Chang, MD, PhD Division of Chest Medicine, Department of Internal Medicine, Taichung Veterans General Hospital, No. 1650, Sect. 4, Taiwan Boulevard, Taichung, 407 Taiwan, R.O.C.

Tel: 886-4-23592525 (ext. 3200)

Fax: 886-4-23741320

E-mail: august@vghtc.gov.tw

Co-correspondence: Tsung-Ying Yang, MD, PhD Faculty of Medicine, School of Medicine,

National Yang-Ming University, No. 155, Sect. 2,

Linong Street, Taipei 11221, Taiwan, R.O.C

Tel: 886-4-23592525 (ext. 3219)

Fax: 886-4-23741320

E-mail: jonyin@gmail.com

Received October 25, 2017

Accepted January 2, 2018

Published Online January 4, 2018

*Yen-Hsiang Huang and Kuo-Hsuan Hsu contributed equally to this work.

\section{Purpose}

The main objective of this study was to investigate the relationship among the clinical characteristics and the frequency of T790M mutation in advanced epidermal growth factor receptor (EGFR) - mutant lung adenocarcinoma patients with acquired resistance after firstline EGFR-tyrosine kinase inhibitor (TKI) treatment.

\section{Materials and Methods}

We enrolled EGFR-mutant stage IIIB-IV lung adenocarcinoma patients, who had progressed to prior EGFR-TKI therapy, and evaluated their rebiopsy EGFR mutation status.

\section{Results}

A total of 205 patients were enrolled for analysis. The overall T790M mutation rate of rebiopsy was 46.3\%. The T790M mutation rates among patients with exon 19 deletion mutation, exon 21 L858R point mutation, and other mutations were 55.0\%, 37.3\%, and $27.3 \%$, respectively. Baseline exon 19 deletion was associated with a significantly higher frequency of T790M mutation (adjusted odds ratio, 2.14; 95\% confidence interval [Cl], 1.20 to 3.83; $p=0.010$ ). In the exon 19 deletion subgroup, there was a greater prevalence of T790M mutation than other exon 19 deletion subtypes in patients with the Del E746-A750 mutation (61.6\% vs. $40.6 \%$; odds ratio, $2.35 ; 95 \% \mathrm{Cl}, 1.01$ to $5.49 ; \mathrm{p}=0.049)$. The progression-free survival (PFS) of first-line TKI treatment $>11$ months was also associated with a higher T790M mutation rate (54.1\% vs. 39.3\%; adjusted odds ratio, 1.82; $95 \% \mathrm{Cl}, 1.02$ to 3.25; $\mathrm{p}=0.044)$. Patients who underwent rebiopsy at metastatic sites had more chance to harbor T790M mutation (52.6\% vs. 33.8\%; adjusted odds ratio, 1.97; 95\% Cl, 1.06 to 3.67; $\mathrm{p}=0.032)$.

\section{Conclusion}

PFS of first-line EGFR-TKI, rebiopsy site, EGFR exon 19 deletion and its subtype Del E746A750 mutation are associated with the frequency of T790M mutation.

\author{
Key words \\ Lung neoplasms, Adenocarcinoma, \\ Epidermal growth factor receptor mutation, \\ T790M, Exon 19 deletion
}




\section{Introduction}

Lung cancer is the leading cause of cancer-related death worldwide. Non-small cell lung cancer (NSCLC) accounts for about $80 \%-85 \%$ of all lung cancer. The treatment of lung cancer is individualized according to the results of molecular biology tests and histology. Epidermal growth factor receptor $(E G F R)$ mutation is the most common driver mutation gene among lung cancer patients in East Asians, as well in Taiwan [1,2]. Previous clinical trials and studies had demonstrated that EGFR-tyrosine kinase inhibitors (TKI) had a better response rate and less adverse effect than platinum-based chemotherapy in EGFR-mutant NSCLC patients [3-9].

Although most EGFR-mutant NSCLC patients have a good response to EGFR-TKI initially, progressive disease (PD) eventually occurs due to acquired resistance. Various mechanisms of acquired resistance to first- and second-generation EGFR-TKIs have been identified, including secondary EGFR mutation, which involves a substitution of threonine to methionine at position 790 (T790M), small cell transformation, mesenchymal-epithelial transition (MET) amplification, epithelial-to-mesenchymal transition, phosphatidylinositol4,5-bisphosphate 3-kinase catalytic subunit alpha (PIK3CA) mutations, and so on [10-12]. Among the above mechanisms, the T790M mutation accounts for $50 \%-60 \%$ of acquired resistance mechanisms $[10,13]$.

The third-generation of EGFR-TKI had significantly greater efficacy than standard chemotherapy with platinum plus pemetrexed in advanced T790M-positive NSCLC patients who had PD after first-line EGFR-TKI treatment [14-16]. Thus, rebiopsy was needed to document the mechanism of acquired resistance for EGFR-mutant patients with PD after EGFR-TKI treatment. Although the mechanisms are yet to be fully elucidated, the clinical predictors for T790M mutation are important. Recent studies have discussed the association between exon 19 deletion (19Del) at baseline and the frequency of T790M mutation [17-19]. However, the results were not consistent and the studies had relatively low patient numbers.

Our previous study indicated that rebiopsy timing did not influence the detection rate of T790M and that the mutation could be identified in patients with a long EGFR-TKI-free interval [20]. Also, in that study, we did not fully explore the associations between patients' clinical characteristics and the prevalence of T790M mutation in mixed lines of EGFR-TKI treatment. Therefore, in this study, we limited the scope of our investigation to advanced lung adenocarcinoma patients with acquired resistance after first-line EFGF-TKI treatment. The main objective of this study was to investigate the relationship among the clinical characteristics, EGFR mutation subtypes, and the frequency of T790M mutation.

\section{Materials and Methods}

\section{Patients}

This study was a retrospective, single-center, observational study at Taichung Veterans General Hospital (TCVGH) in Taiwan. We enrolled lung cancer patients who received rebiopsy between July 2014 and August 2017. To be eligible for the study, patients had to fulfill the inclusion criteria as follows: histologically and cytologically confirmed EGFRmutant lung adenocarcinoma, stage IIIB-IV, according to the seventh edition of the American Joint Committee for Cancer staging system [21], first-line EGFR-TKI treatment, rebiopsy after disease progression confirmed by the Response Evaluation Criteria in Solid Tumors ver. 1.1 [22]. Patients were excluded if they had primary treatment failure to EGFR-TKI, if the T790M mutation presented before EGFR-TKI treatment, or if they had been diagnosed with another active malignancy. Demographic characteristics and clinical data, including age, sex, smoking status, baseline EGFR mutation status, the type of first-line EGFR-TKI treatment, progression-free survival (PFS) of first-line EGFR-TKI, and rebiopsy site were collected for analysis. A never-smoker was defined as someone who had never smoked or smoked less than 100 cigarettes in his or her lifetime.

\section{EGFR mutation test}

EGFR mutations were assessed by matrix-assisted laser desorption ionization-time of flight mass spectrometry, as described in our previous study [2]. All tests were performed at the ISO15189-certified TR6 Pharmacogenomics Laboratory, as part of the National Research Program for Biopharmaceuticals, in the National Center of Excellence for Clinical Trial and Research of National Taiwan University Hospital.

\section{Statistical analyses}

Univariate analysis by Fisher exact test was performed for assessing the associations between the frequency of T790M mutation and patients' characteristics, including age, sex, smoking status, baseline EGFR mutation status, the type of first-line EGFR-TKI treatment, PFS of first-line EGFR-TKI, and rebiopsy site. Multivariate analysis for the impact of baseline EGFR mutation status, PFS of first-line EGFR-TKI and rebiopsy site to the T790M mutation of rebiopsy was performed by logistic regression models. All statistical tests were done with SPSS ver. 23.0 (IBM Corp., Armonk, NY). Two-tailed tests and $\mathrm{p}$-values of $<0.05$ for significance were used. 


\section{Ethical statement}

The study was approved by the Institutional Review Board (IRB) of TCVGH, Taiwan, and written informed consent for genetic testing and clinical data records were obtained from all patients (IRB No. CF12019).

\section{Results}

\section{Patients' characteristics}

In total, 205 patients with advanced EGFR mutant lung adenocarcinoma met the study criteria and were enrolled for analysis (Fig. 1). The baseline characteristics are shown in Table 1 . The median age of the patients was 60 years (range, 32 to 88 years). Seventy-six patients were male (37.1\%), and 129 patients were female (62.9\%). Most of the patients were never-smokers $(\mathrm{n}=162,79.0 \%)$, and 43 patients were former or current smokers (21.0\%). Regarding baseline EGFR mutation status, 111 patients harbored the exon 19 deletion mutation (19Del) (54.1\%), 83 patients harbored the exon $21 \mathrm{~L} 858 \mathrm{R}$ point mutation (L858R) (40.5\%), and 11 patients had other mutations including mixed mutation (5.4\%) (one patient with 19Del+L858R, one patient with 19Del+G719S, one patient with G719S+S768I, one patient with G719A+S861I, one patient with G719A+L861Q, two patients with G719S+L861Q, and four patients with L861Q). Ninety-four patients used gefitinib (45.9\%) as the first-line EGFR-TKI treatment, and 98 patients were treated with erlotinib (47.8\%). Concerning the best response of first-line EGFR-TKI, 176 patients had a partial response $(85.9 \%)$, whereas 29 patients had stable disease (14.1\%). With respect to the PFS of first-line EGFR-TKI, 98 patients had PFS $>11$ months $(47.8 \%)$, and 107 patients had PFS $\leq 11$ months (52.2\%). Rebiopsy of the primary tumor was performed in 68 patients (33.2\%), and rebiopsy of metastatic lesions was performed in 137 patients $(66.8 \%)$.

\section{The T790M mutation status of rebiopsy}

The results of T790M mutation status of rebiopsy and baseline EGFR mutation status are summarized in Fig. 2. The overall T790M mutation rate of rebiopsy was $46.3 \%$. According to the baseline EGFR mutation status, 61 of 111 patients with the 19Del mutation had the T790M mutation (55.0\%), 31 of 83 patients with the L858R mutation harbored the T790M mutation (37.3\%), and three of 11 patients with other mutations (19Del+L858R, 19Del+G719S, and G719S+S768I) tested positive for the T790M mutation (27.3\%).

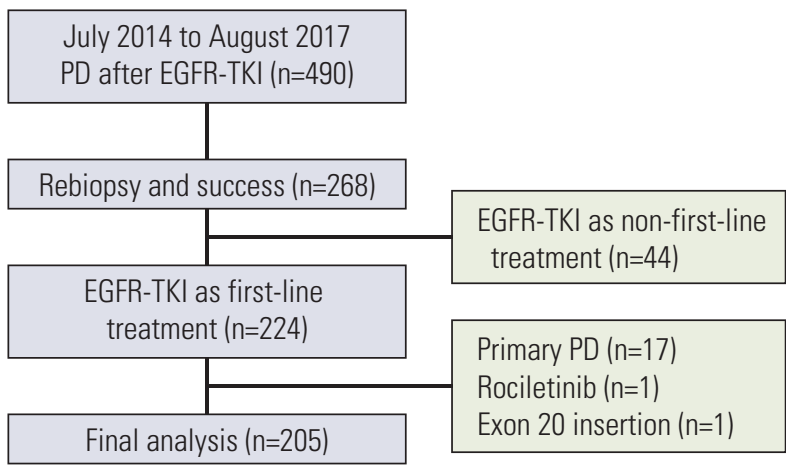

Fig. 1. The patient collection flow chart. PD, progressive disease; EGFR, epidermal growth factor receptor; TKI, tyrosine kinase inhibitor.

Table 1. Patients' characteristics and demographic data

\begin{tabular}{|c|c|}
\hline Characteristic & No. $(\%)(n=205)$ \\
\hline Age, median (range, yr) & $60(32-88)$ \\
\hline \multicolumn{2}{|l|}{ Sex } \\
\hline Male & $76(37.1)$ \\
\hline Female & $129(62.9)$ \\
\hline \multicolumn{2}{|l|}{ Smoking status } \\
\hline Never smokers & $162(79.0)$ \\
\hline Former smokers & $23(11.2)$ \\
\hline Current smokers & $20(9.8)$ \\
\hline \multicolumn{2}{|c|}{ Baseline EGFR mutation status } \\
\hline Exon 19 deletions & $111(54.1)$ \\
\hline Exon 21 L858R & $83(40.5)$ \\
\hline Other mutations $\mathrm{s}^{\mathrm{a})}$ & $11(5.4)$ \\
\hline \multicolumn{2}{|l|}{ First-line TKI } \\
\hline Gefitinib & $94(45.9)$ \\
\hline Erlotinib & $98(47.8)$ \\
\hline Afatinib & $13(6.3)$ \\
\hline \multicolumn{2}{|c|}{ Best response of first-line TKI } \\
\hline Stable disease & $29(14.1)$ \\
\hline Partial response & $176(85.9)$ \\
\hline \multicolumn{2}{|l|}{ PFS of first-line TKI } \\
\hline$\leq 11 \mathrm{mo}$ & $107(52.2)$ \\
\hline$>11 \mathrm{mo}$ & $98(47.8)$ \\
\hline \multicolumn{2}{|l|}{ Rebiopsy site } \\
\hline Primary tumor & $68(33.2)$ \\
\hline Metastases & $137(66.8)$ \\
\hline
\end{tabular}

EGFR, epidermal growth factor receptor; TKI, tyrosine kinase inhibitor; PFS, progression-free survival. a)Includes complex mutation. 


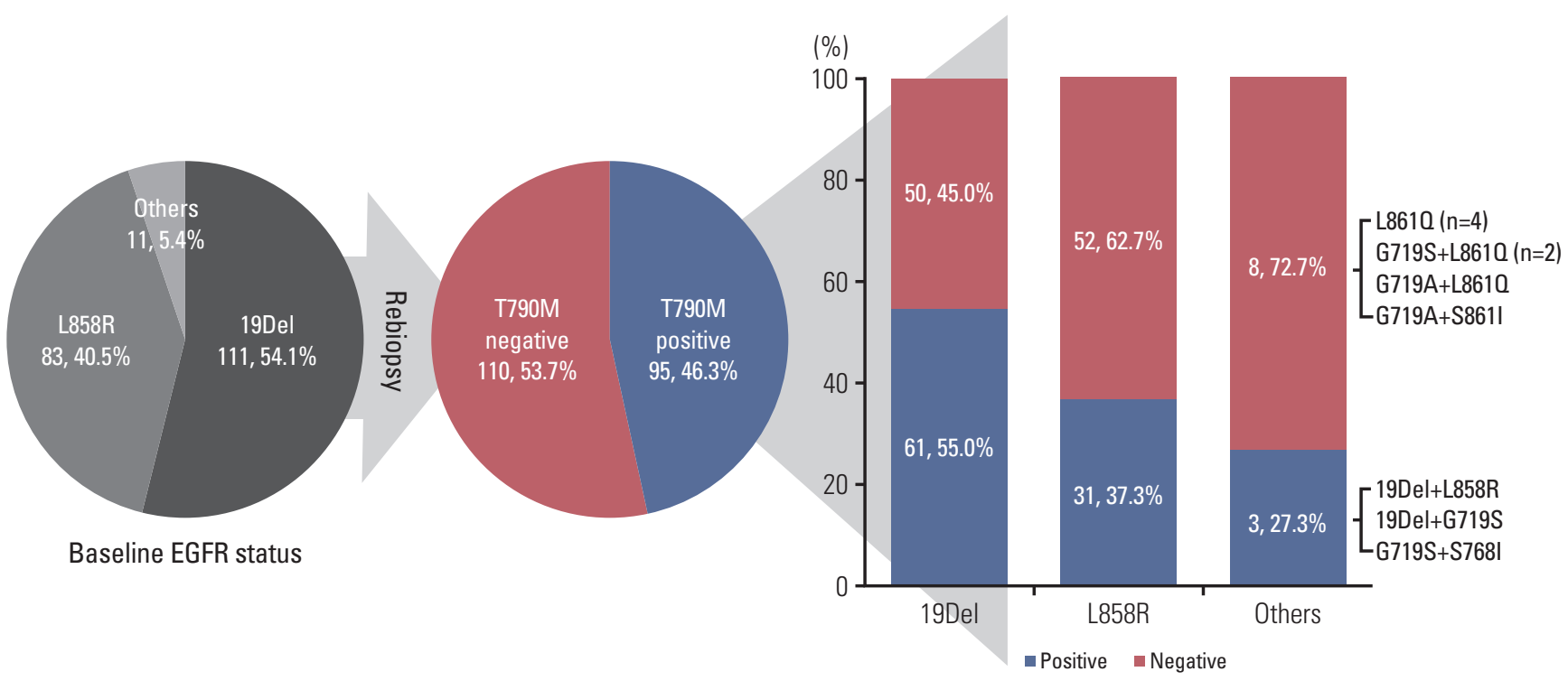

Fig. 2. The association between baseline epidermal growth factor receptor (EGFR) mutation status and T790M mutation status of rebiopsy.

\section{The association between T790M mutation status of rebiopsy and patients' characteristics}

Univariate analyses of the associations between T790M mutation status and patients' characteristics are summarized in Table 2. Patients with baseline 19Del mutation had a significantly higher positive rate of T790M mutation than patients with L858R or other mutations ( $\mathrm{p}=0.022$ ). Patients with a PFS of first-line EGFR-TKI $>11$ months harbored a higher frequency of T790M mutation than that of patients with a PFS of first-line EGFR-TKI $\leq 11$ months $(p=0.036$ ). Patients who underwent rebiopsy at metastatic site had higher incidence of $7790 \mathrm{M}$ mutation than at primary site $(p=0.012)$. There were no relationships of T790M mutation status of rebiopsy with age, sex, smoking status, the type of first-line EGFR-TKI treatment and best response of first-line EGFR-TKI. The detail of relationship between metastatic sites and the frequency of T790M mutation was presented in S1 Table. Additionally, we showed the rebiopsy methods in S2 Table.

\section{The association between T790M mutation status of rebiopsy and exon 19 deletion}

Not only univariate analysis but also multivariate analysis by logistic regression models demonstrated a significantly higher T790M mutation rate in patients with 19Del (adjusted odds ratio, 2.14; 95\% confidence interval [CI], 1.20 to 3.83; $\mathrm{p}=0.010$ ) (Table 3). Because the frequency of the T790M mutation was significantly different between patients with the baseline 19Del mutation and patients with other mutations, we performed a further detailed analysis of the relationship between the subtype of 19Del and T790M mutation of rebiopsy (Table 4). Six patients with the baseline 19Del did not have subtype data. Thus, only 105 patients were enrolled in the analysis. Seventy-three patients had the Del E746-A750 mutation, and the positive rate of T790M mutation was $61.6 \%$. According to the univariate analysis, patients with the Del E746-A750 mutation had a significantly higher frequency of T790M mutation than that of patients with other mutations (odds ratio, 2.35; 95\% CI, 1.01 to 5.49; $\mathrm{p}=0.049$ ), and there was a trend by multivariate analysis (adjusted odds ratio, 2.37; 95\% CI, 0.96 to $5.87 ; \mathrm{p}=0.062$ ).

\section{The association between T790M mutation status of rebiopsy and PFS of first-line EGFR-TKI}

Univariate analysis demonstrated patients with PFS of first-line EGFR-TKI > 11 months harbored a higher frequency of the T790M mutation ( $\mathrm{p}=0.036$ ) (Table 2). Multivariate analysis by logistic regression models showed patients with PFS > 11 months after first-line EGFR-TKI treatment had a higher T790M mutation positive rate with an adjusted odds ratio of 1.82 (95\% CI, 1.02 to $3.25 ; p=0.044)$ (Table 3$)$. We divided patients into three groups (PFS: $\leq 11$ months, 11-20 months, and $>20$ months), and it appeared that the frequency of T790M mutation and PFS of first-line EGFR-TKI treatment were directly correlated (Fig. 3). 
Table 2. Univariate analysis of the association between patients' characteristics and T790M status of rebiopsy

\begin{tabular}{|c|c|c|c|c|}
\hline \multirow{2}{*}{ Characteristic } & \multirow{2}{*}{ No. } & \multicolumn{2}{|c|}{ T790M status } & \multirow{2}{*}{ p-value ${ }^{a}$} \\
\hline & & Positive & Negative & \\
\hline \multicolumn{5}{|l|}{ Age (yr) } \\
\hline$\leq 60$ & 103 & $51(49.5)$ & $52(50.5)$ & 0.402 \\
\hline$>60$ & 102 & $44(43.1)$ & $58(56.9)$ & \\
\hline \multicolumn{5}{|l|}{ Sex } \\
\hline Male & 76 & $33(43.4)$ & $43(56.6)$ & 0.563 \\
\hline Female & 129 & $62(48.1)$ & $67(51.9)$ & \\
\hline \multicolumn{5}{|l|}{ Smoking status } \\
\hline Non-smokers & 162 & $79(48.8)$ & $83(51.2)$ & 0.229 \\
\hline Current/Former-smokers & 43 & $16(37.2)$ & $27(62.8)$ & \\
\hline \multicolumn{5}{|l|}{ Baseline EGFR mutation status } \\
\hline 19Del & 111 & $61(55.0)$ & $50(45.0)$ & 0.022 \\
\hline L858R & 83 & $31(37.3)$ & $52(62.7)$ & \\
\hline Others & 11 & $3(27.3)$ & $8(72.7)$ & \\
\hline \multicolumn{5}{|l|}{ First-line TKI } \\
\hline Gefitinib & 94 & $47(50.0)$ & $47(50.0)$ & 0.188 \\
\hline Erlotinib & 98 & $45(45.9)$ & $53(54.1)$ & \\
\hline Afatinib & 13 & $3(23.1)$ & $10(76.9)$ & \\
\hline \multicolumn{5}{|l|}{ Best response of first line TKI } \\
\hline Stable disease & 29 & $12(41.4)$ & $17(58.6)$ & 0.688 \\
\hline Partial response & 176 & $83(47.2)$ & $93(52.8)$ & \\
\hline \multicolumn{5}{|l|}{ PFS of first-line TKI } \\
\hline$\leq 11 \mathrm{mo}$ & 107 & $42(39.3)$ & $65(60.7)$ & 0.036 \\
\hline$>11 \mathrm{mo}$ & 98 & $53(54.1)$ & $45(45.9)$ & \\
\hline \multicolumn{5}{|l|}{ Rebiopsy site } \\
\hline Primary & 68 & $23(33.8)$ & $45(66.2)$ & 0.012 \\
\hline Metastases & 137 & $72(52.6)$ & $65(47.4)$ & \\
\hline
\end{tabular}

Values are presented as number (\%). EGFR, epidermal growth factor receptor; TKI, tyrosine kinase inhibitor; PFS, progression-free survival. ${ }^{\text {a) }}$ B Fisher exact test.

Table 3. Multivariate analysis of the association between patients' characteristics and T790M status of rebiopsy

\begin{tabular}{|c|c|c|c|c|}
\hline Characteristic & Odds ratio $(95 \% \mathrm{CI})$ & p-value ${ }^{a}$ & Adjusted odds ratio (95\% CI) & p-value $e^{a}$ \\
\hline \multicolumn{5}{|c|}{ Baseline EGFR mutation status } \\
\hline 19Del vs. others & $2.15(1.23-3.78)$ & 0.008 & $2.14(1.20-3.83)$ & $0.010^{\mathrm{b})}$ \\
\hline \multicolumn{5}{|l|}{ PFS of first-line TKI } \\
\hline$>11 \mathrm{mo}$ vs. $\leq 11 \mathrm{mo}$ & $1.82(1.05-3.18)$ & 0.034 & $1.82(1.02-3.25)$ & $0.044^{c)}$ \\
\hline \multicolumn{5}{|l|}{ Rebiopsy site } \\
\hline Metastasis vs. primary & $2.17(1.19-3.97)$ & 0.012 & $1.97(1.06-3.67)$ & $0.032^{\mathrm{d})}$ \\
\hline
\end{tabular}

CI, confidence interval; EGFR, epidermal growth factor receptor; PFS, progression-free survival; TKI, tyrosine kinase inhibitor.

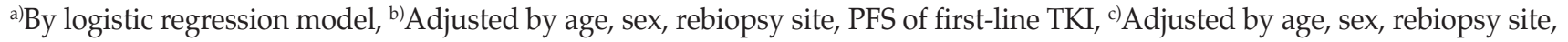
and baseline EGFR mutation status, d)Adjusted by age, sex, PFS of first-line TKI and baseline EGFR mutation status. 
Table 4. EGFR exon 19 deletions mutation patterns in relation to T790M status of rebiopsy ${ }^{\text {a) }}$

\begin{tabular}{lrrr} 
& & \multicolumn{2}{c}{ T790M status } \\
\cline { 2 - 3 } Exon 19 deletions type & No. & Positive & Negative \\
Del E746-A750 & 73 & $45(61.6)$ & $28(38.4)$ \\
Del L747-P753 ins S & 7 & $4(57.1)$ & $3(42.9)$ \\
Del E746-S752 ins V & 6 & $1(16.7)$ & $5(83.3)$ \\
Del E746-T751 & 3 & $1(33.3)$ & $2(66.7)$ \\
Del L747-T751 & 2 & $1(50.0)$ & $1(50.0)$ \\
Del L747-T751 ins P & 2 & 0 & $2(100)$ \\
Del L747-E750 & 2 & 0 & $2(100)$ \\
Del L747-A750 & 1 & 0 & $1(100)$ \\
Del L747-P753 & 1 & 0 & $1(100)$ \\
Mixed & 8 & $6(75.0)$ & $2(25.0)$ \\
Total & 105 & 58 & 47 \\
\hline
\end{tabular}

Values are presented as number $(\%)$. EGFR, epidermal growth factor receptor. ${ }^{\text {a) }}$ Six patients without subtype data.

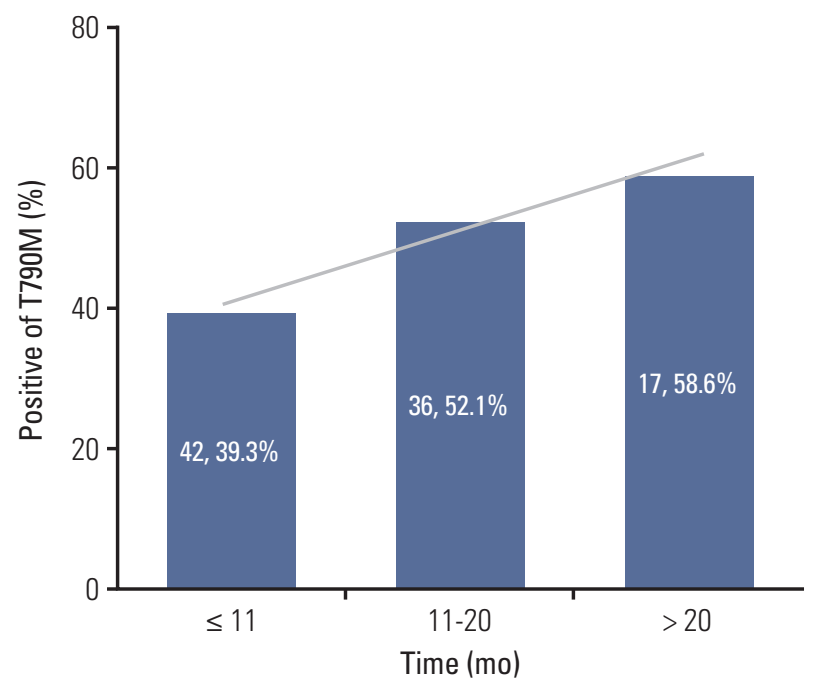

Fig. 3. The association between progression-free survival of first-line epidermal growth factor receptor-tyrosine kinase inhibitor and T790M mutation status of rebiopsy.

The analysis of the major mutations (exon 19 deletion mutation and exon $21 \mathrm{~L} 858 \mathrm{R}$ point mutation) revealed that the median PFS of first-line EGFR-TKI was 11.5 months in patients with the T790M mutation, and 9.1 months in patients without the T790M mutation. There was no statistically significant relationship between PFS and the T790M mutation status in patients harboring the exon 19 deletion and the L858R mutation ( $\mathrm{p}=0.235)$ (Fig. 4A). Additionally, we divided the patients into four groups to evaluate the association between PFS of first-line EGFR-TKI therapy, baseline EGFR mutation status, and T790M mutation status of rebiopsy (19Del with T790M, 19Del without T790M, L858R with T790M, L858R without T790M), and no significant differences in PFS was found among these four groups $(\mathrm{p}=0.250)$ (Fig. 4B).

\section{The association between clinical characteristics and the first-line EGFR-TKI treatment}

We showed the relationship between clinical characteristics and the first-line EGFR-TKI in S3 Table. Fifty-three patients $(54.1 \%)$ were female in erlotinib group, and seven patients $(53.8 \%)$ were female in afatinib group. Sixty-nine patients $(73.4 \%)$ were female in gefitinib group, and significant higher female percentage than other groups was noted by univariate analysis $(p=0.015)$. There were no difference of age, smoking status, baseline EGFR mutation status, best response of first-line EGFR-TKI, PFS of first-line EGFR-TKI and rebiopsy site between three kinds of EGFR-TKI.

\section{Discussion}

Our study demonstrated that PFS of first-line EGFR-TKI $>11$ months and rebiopsy at metastatic sites were correlated with a higher frequency of the T790M mutation. More importantly, not only the EGFR exon 19 deletion but also its subtype deletion E746-A750 at baseline was a predictor of the T790M mutation.

Nosaki et al. [17] stated that the frequency of the T790M mutation was higher in patients with the EGFR exon 19 dele- 
A
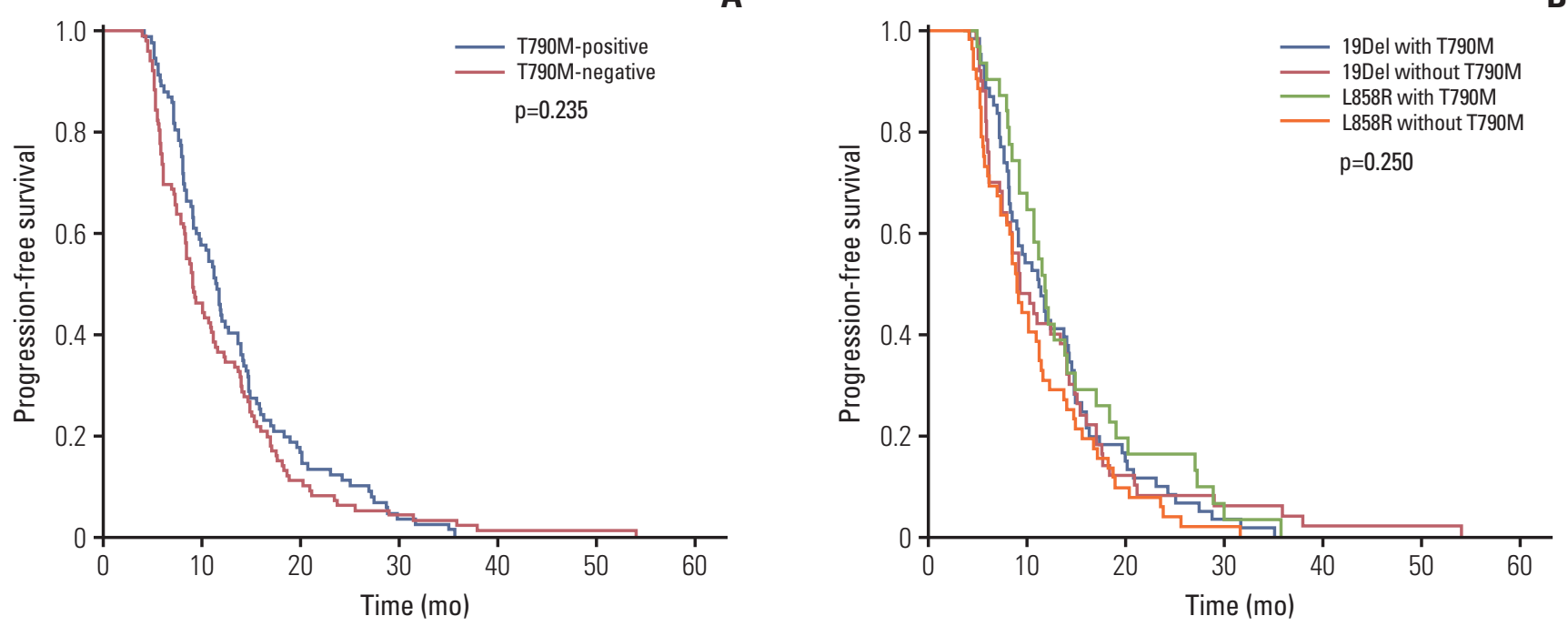

Fig. 4. (A) Progression-free survival (PFS) of first-line epidermal growth factor receptor (EGFR)-tyrosine kinase inhibitor (TKI) versus T790M mutation status of rebiopsy by Kaplan-Meier survival curve analysis. (B) PFS of first-line EGFR-TKI versus T790M mutation status of rebiopsy and baseline EGFR mutation status by Kaplan-Meier survival curve analysis.

tion than in those with the L858R mutation (55.6\% vs. 43.0\%, $\mathrm{p}=0.05$ by Fisher exact test). Matsuo et al. [18] also reported that patients with the 19Del mutation had a higher frequency of the T790M mutation than patients with the L858R mutation (63\% vs. 38\%, p=0.035). However, Oya et al. [19] demonstrated that there was no relationship between the baseline EFGR mutation and T790M mutation status (exon 19 deletion $49.5 \%$, L858R $48.1 \%, \mathrm{p}=0.240$ ). Because the results were inconsistent, we designed the present study to include a relatively homogenous patient population and a larger total number of patients. We enrolled only adenocarcinoma patients who had acquired resistance after first-line EGFRTKI treatment. Patients with primary T790M and patients who failed to respond to primary treatment with EGFR-TKI were excluded. We then compared the frequency of EGFR mutation between patients with 19Del and those with other mutations. A significantly higher T790M mutation positive rate in patients with 19Del was found in both the univariate and multivariate analysis. Additionally, in the present study, three patients with a complex EGFR mutation had the T790M mutation after first-line EGFR-TKI treatment. Two of them had the exon 19 deletion plus another mutation (19Del+ L858R and 19Del+G719S). It appeared that the baseline 19Del was still the most influential mutation type.

Regarding the subtypes of EGFR exon 19 deletions, the most common subtype in our study was Del E746-A750, followed by Del L747-P753 ins S, which was consistent with the results of previous study [23,24]. Lee et al. [23] disclosed that patients with the exon 19 deletion starting on codon E746 had a better median PFS than those starting on L747 (14.2 months vs.6.5 months, $\mathrm{p}=0.021$ ). Chung et al. [24] reported patients with LRE deletions in exon 19 had a better response to EGFRTKIs, compared to patients with non-LRE deletions. In the present study, we evaluated the relationship between the subtype of baseline 19Del mutation and T790M mutation status after acquired resistance to first-line EGFR-TKI. Univariate analysis showed that patients with the Del E746-A750 mutation had a higher frequency of the T790M mutation than patients with other subtypes, and it had a trend by multivariate analysis also. This is the first study to demonstrate the association between the subtype Del E746-A750 of EGFR exon 19 deletion and positive T790M status.

Structural analyses showed an allosteric mechanism important for EGFR kinase domain activation in a previous report [25]. The deletions in EGFR exon 19, located between strand $\beta 3$ and helix $\alpha \mathrm{C}$, could disrupt inactive conformation of the EGFR kinase domain and enhance the effectiveness of EGFR-TKIs [26]. However, because the deletions can occur in various fragments of exon 19 of the EGFR gene, the exon 19 deletion mutation is relatively complex compared with other EGFR mutations. Thus, it is challenging to elucidate the precise mechanism by which the pathophysiology of the subtype of exon19 deletion influences the frequency of the T790M mutation. Further research is needed to understand the underlying mechanisms involved.

Concerning the PFS of first-line EGFR-TKI treatment, Matsuo et al. [18] stated that the total duration of EGFR-TKI treatment was related to the frequency of patients with T790M 


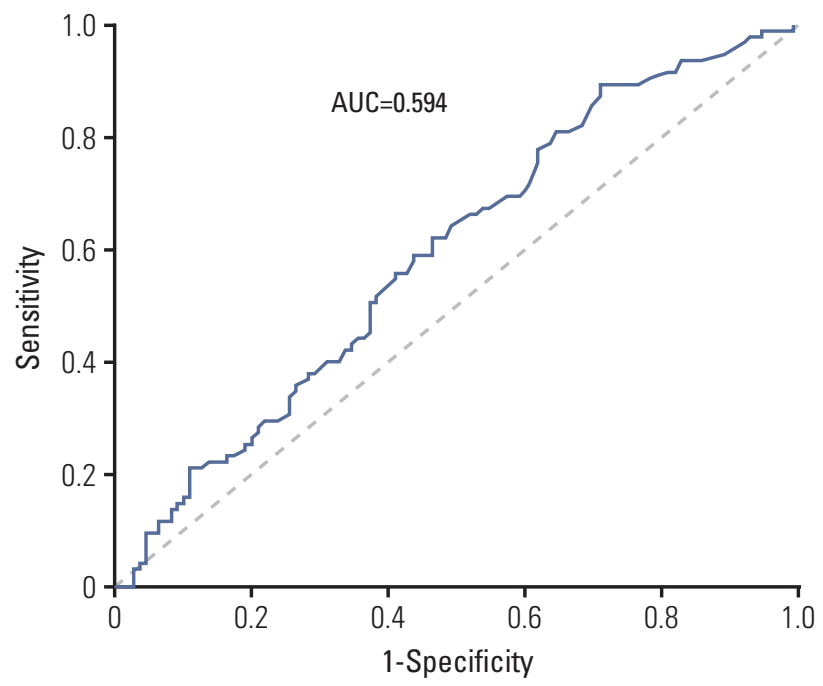

Fig. 5. The relationship between the duration of progression-free survival and T790M detection rate by receiver operating characteristic curve analysis. AUC, area under curve.

mutation. Oya et al. [19] demonstrated patients with PFS less than 6 months were significantly less likely to harbor the T790M mutation than those with PFS $\geq 6$ months. In our study, the median PFS was 10.7 months, and we found that patients with PFS > 11 months under first-line EGFR-TKI therapy had a higher prevalence of T790M mutation than patients with PFS $\leq 11$ months. Although the area under curve in the receiver operating characteristic curve analysis of the association between the duration of PFS and T790M detection rate was only 0.594 (Fig. 5), we found there was a direct correlation between the PFS of first-line EGFR-TKI and the frequency of T790M mutation, as shown in Fig. 3. The above research may support the concept that longer EGFRTKIs exposure induces a greater frequency of T790M mutation.

Moreover, previous studies demonstrated that the PFS of initial EGFR-TKI treatment was longer in patients with the T790M mutation $[18,19]$. In our study, the median PFS of first-line EGFR-TKI was 11.5 months in patients with the T790M mutation, and 9.1 months in patients without the T790M mutation. There was no statistically significant relationship between PFS and T790M mutation status ( $\mathrm{p}=0.235$ ). In the present study, we excluded patients who failed to respond to primary treatment with EGFR-TKIs, while other studies usually included patients with primary treatment failure. Patients with primary PD usually had a lower rate of T790M compared with patients with acquired resistance [19]. Patients with primary PD had a shorter PFS than patients with acquired resistance, which explains why there was no statistically significant result despite a difference in PFS of 2.4 months in this study. These findings are consistent with the idea that patients who acquired the T790M mutation have more indolent tumors than those without the T790M mutation. Compared with other studies, our patient group was relatively homogenous, had a higher number of patients, and our investigation was more clinically oriented.

Additionally, our study demonstrated a significantly higher T790M mutation positive rate in patients who received rebiopsy at metastatic site by both univariate and multivariate analysis (adjusted odds ratio, 1.97; 95\% CI, 1.06 to 3.67; $\mathrm{p}=0.032$ ). This could be explained by the tumor heterogeneity and the different characteristics between primary tumor and metastatic lesions $[27,28]$. Previous studies had suggested that tissue specimen should be obtained on new or progressing lesions after disease progression to determine the suitable treatments against the EGFR mutation profiles $[28,29]$.

Concerning the relationship between prior EGFR-TKI treatment and the frequency of T790M mutation, patients who treated with afatinib as prior TKI had lower positive rate of T790M mutation $(3 / 13,23.1 \%)$ than gefitinib group $(47 / 94,50.0 \%)$ and erlotinib group $(45 / 98,45.9 \%)$ in present study. According to previous studies, the range of frequency of T790M mutation was from $25 \%$ to $47.6 \%$ [18,19,30,31]. However, the patient number and patient group were heterogeneous. We included only 13 patients with afatinib treatment, and it was difficult to make conclusion about the exact T790M mutation rate due to smaller patient numbers.

Our study had some limitations. First, it was a retrospective study, and thus more bias may have been present compared with a study that had been prospectively designed. Second, the research was undertaken at a single center, and therefore there may have been selection bias. Third, all patients in this study were Taiwanese, so our findings may not be generalizable to other ethnic populations. Although this study is the first to discuss the relationship between the subtypes of baseline 19Del and the frequency of T790M mutation status after acquired resistance to first-line EGFRTKI treatment, further research is needed to confirm our results.

Our findings shed light on which patients may have a greater tendency to develop the T790M mutation after EGFRTKIs treatment. Our study demonstrated that PFS of firstline EGFR-TKI and rebiopsy site influence the frequency of the T790M mutation. More importantly, both the exon 19 deletion and its subtype at baseline were predictors of the T790M mutation. 


\section{Electronic Supplementary Material}

Supplementary materials are available at Cancer Research and Treatment website (http:// www.e-crt.org).

\section{Conflicts of Interest}

Conflict of interest relevant to this article was not reported.

\section{Acknowledgments}

We would like to thank the Comprehensive Cancer Center of Taichung Veterans General Hospital for its assistance with the collection and management of the data.

\section{Author Details}

${ }^{1}$ Division of Chest Medicine, Department of Internal Medicine and 2Division of Critical Care and Respiratory Therapy, Department of Internal Medicine, Taichung Veterans General Hospital, Taichung, ${ }^{3}$ Institute of Biomedical Sciences, National Chung Hsing University, Taichung, 'Faculty of Medicine, School of Medicine, National YangMing University, Taipei, ${ }^{5}$ Department of Clinical Laboratory Sciences and Medical Biotechnology, National Taiwan University College of Medicine, Taipei, ${ }^{6}$ Department of Laboratory Medicine, National Taiwan University Hospital, Taipei, ${ }^{7}$ Graduate Institute of Toxicology, National Taiwan University, Taipei, ${ }^{8}$ Center of Genomic Medicine, National Taiwan University College of Medicine, Taipei, ${ }^{9}$ Department of Pathology and Graduate Institute of Pathology, National Taiwan University College of Medicine, Taipei, ${ }^{10}$ Center for Optoelectronic Biomedicine, National Taiwan University College of Medicine, Taipei, ${ }^{11}$ Comprehensive Cancer Center, Taichung Veterans General Hospital, Taichung, Taiwan

\section{References}

1. Shi Y, Au JS, Thongprasert S, Srinivasan S, Tsai CM, Khoa MT, et al. A prospective, molecular epidemiology study of EGFR mutations in Asian patients with advanced non-small-cell lung cancer of adenocarcinoma histology (PIONEER). J Thorac Oncol. 2014;9:154-62.

2. Hsu KH, Ho CC, Hsia TC, Tseng JS, Su KY, Wu MF, et al. Identification of five driver gene mutations in patients with treatment-naive lung adenocarcinoma in Taiwan. PLoS One. 2015;10:e0120852.

3. Zhou C, Wu YL, Chen G, Feng J, Liu XQ, Wang C, et al. Erlotinib versus chemotherapy as first-line treatment for patients with advanced EGFR mutation-positive non-smallcell lung cancer (OPTIMAL, CTONG-0802): a multicentre, open-label, randomised, phase 3 study. Lancet Oncol. 2011;12: $735-42$.

4. Rosell R, Carcereny E, Gervais R, Vergnenegre A, Massuti B, Felip E, et al. Erlotinib versus standard chemotherapy as firstline treatment for European patients with advanced EGFR mutation-positive non-small-cell lung cancer (EURTAC): a multicentre, open-label, randomised phase 3 trial. Lancet Oncol. 2012;13:239-46.

5. Maemondo M, Inoue A, Kobayashi K, Sugawara S, Oizumi S, Isobe $\mathrm{H}$, et al. Gefitinib or chemotherapy for non-small-cell lung cancer with mutated EGFR. N Engl J Med. 2010;362: 2380-8.

6. Mitsudomi T, Morita S, Yatabe Y, Negoro S, Okamoto I, Tsurutani J, et al. Gefitinib versus cisplatin plus docetaxel in patients with non-small-cell lung cancer harbouring mutations of the epidermal growth factor receptor (WJTOG3405): an open label, randomised phase 3 trial. Lancet Oncol. 2010;11:
121-8.

7. Sequist LV, Yang JC, Yamamoto N, O'Byrne K, Hirsh V, Mok $\mathrm{T}$, et al. Phase III study of afatinib or cisplatin plus pemetrexed in patients with metastatic lung adenocarcinoma with EGFR mutations. J Clin Oncol. 2013;31:3327-34.

8. Lynch TJ, Bell DW, Sordella R, Gurubhagavatula S, Okimoto RA, Brannigan BW, et al. Activating mutations in the epidermal growth factor receptor underlying responsiveness of nonsmall-cell lung cancer to gefitinib. N Engl J Med. 2004;350: 2129-39.

9. Paez JG, Janne PA, Lee JC, Tracy S, Greulich H, Gabriel S, et al. EGFR mutations in lung cancer: correlation with clinical response to gefitinib therapy. Science. 2004;304:1497-500.

10. Yu HA, Arcila ME, Rekhtman N, Sima CS, Zakowski MF, Pao $\mathrm{W}$, et al. Analysis of tumor specimens at the time of acquired resistance to EGFR-TKI therapy in 155 patients with EGFRmutant lung cancers. Clin Cancer Res. 2013;19:2240-7.

11. Kobayashi S, Boggon TJ, Dayaram T, Janne PA, Kocher O, Meyerson $\mathrm{M}$, et al. EGFR mutation and resistance of nonsmall-cell lung cancer to gefitinib. N Engl J Med. 2005;352: 786-92.

12. Ohashi K, Maruvka YE, Michor F, Pao W. Epidermal growth factor receptor tyrosine kinase inhibitor-resistant disease. J Clin Oncol. 2013;31:1070-80.

13. Sequist LV, Waltman BA, Dias-Santagata D, Digumarthy $S$, Turke AB, Fidias P, et al. Genotypic and histological evolution of lung cancers acquiring resistance to EGFR inhibitors. Sci Transl Med. 2011;3:75ra26.

14. Mok TS, Wu YL, Ahn MJ, Garassino MC, Kim HR, Ramalingam SS, et al. Osimertinib or platinum-pemetrexed in EGFR 
T790M-positive lung cancer. N Engl J Med. 2017;376:629-40.

15. Janne PA, Yang JC, Kim DW, Planchard D, Ohe Y, Ramalingam SS, et al. AZD9291 in EGFR inhibitor-resistant nonsmall-cell lung cancer. N Engl J Med. 2015;372:1689-99.

16. Cross DA, Ashton SE, Ghiorghiu S, Eberlein C, Nebhan CA, Spitzler PJ, et al. AZD9291, an irreversible EGFR TKI, overcomes T790M-mediated resistance to EGFR inhibitors in lung cancer. Cancer Discov. 2014;4:1046-61.

17. Nosaki K, Satouchi M, Kurata T, Yoshida T, Okamoto I, Katakami N, et al. Re-biopsy status among non-small cell lung cancer patients in Japan: a retrospective study. Lung Cancer. 2016;101:1-8

18. Matsuo N, Azuma K, Sakai K, Hattori S, Kawahara A, Ishii H, et al. Association of EGFR exon 19 deletion and EGFR-TKI treatment duration with frequency of T790M mutation in EGFR-mutant lung cancer patients. Sci Rep. 2016;6:36458.

19. Oya Y, Yoshida T, Kuroda H, Shimizu J, Horio Y, Sakao Y, et al. Association between EGFR T790M status and progression patterns during initial EGFR-TKI treatment in patients harboring EGFR mutation. Clin Lung Cancer. 2017;18:698-705.e2.

20. Tseng JS, Su KY, Yang TY, Chen KC, Hsu KH, Chen HY, et al. The emergence of T790M mutation in EGFR-mutant lung adenocarcinoma patients having a history of acquired resistance to EGFR-TKI: focus on rebiopsy timing and long-term existence of T790M. Oncotarget. 2016;7:48059-69.

21. Edge SB, Compton CC. The American Joint Committee on Cancer: the 7th edition of the AJCC cancer staging manual and the future of TNM. Ann Surg Oncol. 2010;17:1471-4.

22. Eisenhauer EA, Therasse P, Bogaerts J, Schwartz LH, Sargent D, Ford R, et al. New response evaluation criteria in solid tumours: revised RECIST guideline (version 1.1). Eur J Cancer. 2009;45:228-47.

23. Lee VH, Tin VP, Choy TS, Lam KO, Choi CW, Chung LP, et al. Association of exon 19 and 21 EGFR mutation patterns with treatment outcome after first-line tyrosine kinase inhibitor in metastatic non-small-cell lung cancer. J Thorac Oncol. 2013;8: 1148-55.

24. Chung KP, Wu SG, Wu JY, Yang JC, Yu CJ, Wei PF, et al. Clinical outcomes in non-small cell lung cancers harboring different exon 19 deletions in EGFR. Clin Cancer Res. 2012;18: 3470-7.

25. Zhang X, Gureasko J, Shen K, Cole PA, Kuriyan J. An allosteric mechanism for activation of the kinase domain of epidermal growth factor receptor. Cell. 2006;125:1137-49.

26. Bose R, Zhang X. The ErbB kinase domain: structural perspectives into kinase activation and inhibition. Exp Cell Res. 2009;315:649-58.

27. Cadranel J, Zalcman G, Sequist L. Genetic profiling and epidermal growth factor receptor-directed therapy in nonsmall cell lung cancer. Eur Respir J. 2011;37:183-93.

28. Jekunen AP. Role of rebiopsy in relapsed non-small cell lung cancer for directing oncology treatments. J Oncol. 2015;2015: 809835.

29. Kim E, Feldman R, Wistuba II. Update on EGFR mutational testing and the potential of noninvasive liquid biopsy in nonsmall-cell lung cancer. Clin Lung Cancer. 2018;19:105-14.

30. Wu SG, Liu YN, Tsai MF, Chang YL, Yu CJ, Yang PC, et al. The mechanism of acquired resistance to irreversible EGFR tyrosine kinase inhibitor-afatinib in lung adenocarcinoma patients. Oncotarget. 2016;7:12404-13.

31. Tanaka K, Nosaki K, Otsubo K, Azuma K, Sakata S, Ouchi H, et al. Acquisition of the T790M resistance mutation during afatinib treatment in EGFR tyrosine kinase inhibitor-naive patients with non-small cell lung cancer harboring EGFR mutations. Oncotarget. 2017;8:68123-30. 